\title{
Identificación bacteriana basada en el espectro de masas de proteínas: Una nueva mirada a la microbiología del siglo XXI
}

\author{
Patricia García, Fidel Allende, Paulette Legarraga, Marcos Huilcaman y Sandra Solari
}

\section{Bacterial identification based on protein mass spectrometry: A new insight at the microbiology of the $21^{\text {st }}$ century}

Bacterial identification is important for the proper treatment of infected patients hospitalized with serious infections especially in critical care units. Identification by conventional methods used in microbiology laboratories takes at least 16 hours since a culture is positive. The introduction of mass spectrometry, specifically MALDI-TOF MS (matrix-assisted laser desorption/ ionization time-of-flight mass spectrometer) in the microbiology laboratory could mean a radical change in the identification accuracy, turn around time (6 minutes per bacteria) and cost (about 5 times cheaper than conventional identification). Since its introduction in clinical microbiology laboratories in 2008, many reports about its usefulness in identifying microorganisms from colonies, as well as directly from positive blood cultures and urine samples have been published. This review describes MALDI-TOF MS methodology, its identification performance for bacteria (aerobic and anaerobic), mycobacterium and yeasts, its future applications in microbiology and its main disadvantages.

Key words: Bacterial identification, mass spectrometry.

Palabras clave: Identificación bacteriana, espectrometría de masas, MALDI-TOF MS.

\section{Introducción}

$\mathrm{T}$ radicionalmente la identificación bacteriana se ha basado en la utilización de métodos fenotípicos que incluyen las características morfológicas y tintoriales que presentan los microorganismos en distintos medios de cultivos, así como las reacciones bioquímicas propias de cada especie ${ }^{1}$. Sin embargo, esto conlleva una carga laboral y consumo de tiempo importante para el laboratorio, puesto que la identificación bacteriana puede tomar desde horas e incluso hasta días, sobre todo para aquellos microorganismos fastidiosos, lo cual sin duda, impacta directamente en el pronóstico del enfermo, pues es sabido que mientras más precoz y apropiado es el antimicrobiano, menor es la mortalidad del paciente, en especial para aquellos que están graves ${ }^{2-4}$. Es así como algunos reportes demuestran un incremento en la mortalidad de $7 \%$ por hora cuando existe un retraso en el inicio de antimicrobianos para aquellas personas que están hipotensas y en estado séptico ${ }^{5}$. Ante esta situación se ha generalizado el uso precoz de antimicrobianos de amplio espectro dado la dificultad para obtener un diagnóstico rápido de las bacterias involucradas, lo que ha llevado al uso, muchas veces, de tratamientos indebidos, con la consiguiente preocupación por la emergencia de bacterias resistentes, la asociación con enfermedades fúngicas invasoras y el costo excesivo de esta política ${ }^{6}$.

Bajo esta inquietud, se han buscado métodos que aumenten la exactitud y disminuyan los tiempos de respuesta en la identificación bacteriana. Entre las técnicas más utilizadas en la microbiología moderna destacan los sistemas en miniatura de pruebas analíticas bioquímicas, cuyo inconveniente es que en algunos casos pueden ser lentos e imprecisos ${ }^{7}$. En esta misma línea están los sistemas automatizados que realizan identificación bacteriana en tiempos más cortos; sin embargo, el costo de implementación es alto ${ }^{8}$. Probablemente lo que ha ganado mayor terreno en la actualidad, es la biología molecular, que si bien es bastante precisa, aún tiene tiempos prolongados de respuesta debido a la falta de automatización universal de sus procesos, lo que la hace susceptible de errores y contaminación; además sus altos costos de implementación la limita a algunos centros.

La identificación bacteriana basada en la espectrometría de masas, específicamente en el MALDI-TOF MS, por su sigla en inglés matrix-assisted laser desorption/ionization time-of-flight mass spectrometer, es una técnica existente hace más de 30 años. No obstante, sólo en el último tiempo ha sido utilizada como un método rápido y acucioso para la identificación de rutina de microorganismos patógenos mediante el perfil de proteínas ${ }^{9,10}$. La espectrometría de masas es un método que permite la identificación de una molécula mediante la medición de su masa en relación a su carga (relación masa/carga), así como la de los fragmentos generados a partir de ella. El primer espectrómetro de masas fue desarrollado en 1912 por J. J. Thomson para el análisis de espectros de elementos como $\mathrm{O}_{2}, \mathrm{~N}_{2}$ y CO. Desde entonces, la evolución en espectrometría de masas ha
Pontificia Universidad Católica de Chile, Santiago. Facultad de Medicina Departamento de Laboratorios Clínicos Laboratorio de Microbiología (PG).

Servicio de Laboratorios Clínicos (MH).

Departamento de Laboratorios Clínicos.

Laboratorio de Bioquímica, Sección Espectrometría de Masas (FA, SS). Departamento de Medicina Interna (PL).

Conflictos de interés: no hay.

Recibido: 19 de julio de 2011 Aceptado: 26 de noviembre de 2011

Correspondencia a: Sandra Solari Gajardo, ssolari@med.puc.cl 
sido muy importante debido a: la asociación con equipos de cromatografía que ha permitido la separación de mezclas de compuestos, previo al análisis de masas, mejorando la selectividad del método y el acoplamiento de múltiples espectrómetros junto al desarrollo de espectrómetros que miden tiempos de migración de las masas, favoreciendo la detección de moléculas de mayor tamaño, como por ejemplo microorganismos completos. La Tabla 1 muestra los diferentes tipos de equipamientos con espectrometría de masas con utilidad en el laboratorio clínico.

El MALDI-TOF MS utiliza el cálculo de tiempo de migración (tiempo de vuelo) de cada fragmento de una molécula a través de un trayecto predeterminado previa desorción/ionización láser de la molécula en una matriz determinada. Este espectrómetro tiene la capacidad de medir macromoléculas de hasta 100.000 Dalton, dentro de las cuales están los péptidos y proteínas que forman parte de hongos y bacterias. Dado que un microorganismo analizado en el MALDI-TOF MS entregará siempre el mismo espectro de masas, los fabricantes de los sistemas MALDI-TOF MS han diseñado archivos con los espectros de masas de la fragmentación de péptidos y proteínas que presentan los distintos microorganismos para una misma emisión del láser y una misma distancia de migración. La identificación se realiza a través de la comparación (correlación) del resultado de una bacteria con todos los espectros de masas que contiene el archivo comercial proporcionado por el fabricante, y de acuerdo a puntos de corte definidos para estas correlaciones. Estos archivos, junto con los software necesarios para su manejo permitieron finalmente el uso de esta herramienta en el campo de la microbiología clínica ${ }^{11}$.

En la actualidad se encuentran disponibles dos instrumentos para el uso en laboratorio de rutina comercia- lizados por Bruker Daltonics Inc. (Billerica, MA, USA) y Shimadzu. Corporation (Kyoto, Japón). En el caso de Bruker se comercializa el espectrómetro junto a la base de datos y software necesarios para la identificación mientras que en el caso de Shimadzu el software es ofrecido por ellos mientras que la base de datos es SARAMIS ${ }^{\mathrm{TM}}$ AnagnosTec (Zossen, Germany)/bioMérieux (Marcy l'Etoile, Francia). Existe otra base de datos llamada Andromas que puede ser utilizada en ambos equipos, la cual contiene información para la identificación de Aspergillus y dermatofitos. Todos los sistemas permiten la conexión a los sistemas informáticos de los laboratorios ${ }^{12}$.

La implementación de MALDI-TOF MS en un laboratorio de microbiología no requiere de infraestructura especial. Los equipos comerciales disponibles en la actualidad no utilizan un espacio mayor al de otros equipos automatizados usados frecuentemente en el laboratorio y tampoco requieren de un ambiente con control de temperatura estricto, a diferencia de otros equipos de espectrometría. Sólo son necesarias una conexión eléctrica y otra conexión a internet. El personal requiere entrenamiento en el equipo y conocimientos básicos de espectrometría de masas $^{13}$.

\section{Metodología}

De manera general, la identificación microbiana por MALDI-TOF MS consta de cuatro pasos: la recuperación de una colonia aislada, la realización de un espectro de masas, la comparación con la base de datos y la entrega de resultados.

La identificación se realiza a partir de una colonia aislada del microorganismo en estudio, la que es aplicada

\section{Tabla 1. Diferentes tipos de equipamientos con espectrometría de masas y su utilidad en laboratorio clínico}

\section{Equipo/método}

Cromatografía gaseosa (GC) con espectrometría de masas (MS)

Cromatografía liquida (LC) con espectrometría de masas (MS)

Cromatografía GC/MS-MS (Tandem)

Cromatografía LC/MS-MS (Tandem)

Detector de masas por tiempo de vuelo con desorción láser asistida por una matriz (MALDI-TOF matrix-assisted desorption ionization-time of flight)

\section{Fundamento}

Permite la separación previa de la mezcla de compuestos presentes en una misma muestra, con un posterior análisis del espectrómetro

Permite la separación de sustancias volátiles y no volátiles

Permite disminuir el tiempo de trabajo en la fase pre analítica en relación al GC/MS

Permite mejorar la sensibilidad y especificidad de los métodos anteriores

Permite mejorar la sensibilidad y especificidad de los métodos anteriores

\section{Consiste en el cálculo de tiempo de vuelo de cada} fragmento de una molécula a través de un trayecto predeterminado con una ionización láser previa de la molécula en una matriz determinada

\section{Utilidad}

Tamaño molecular

Toxicología, medicina forense, laboratorios de alimentos y medioambientales, en general para medición de sustancias volátiles

Laboratorios clínicos (medición de hormonas, tamizaje neonatal de enfermedades metabólicas),

Bioequivalencia, medición de pesticidas

\section{$<1.000 \mathrm{Da}$.}

$<3.000 \mathrm{Da}$

Métodos de referencia para la determinación de $\quad<1.000 \mathrm{Da}$ una gran cantidad de analitos

Métodos de referencia para la determinación de $\quad<3.000 \mathrm{Da}$ una gran cantidad de analitos. Permite análisis de proteínas, aunque la etapa pre-analítica es engorrosa

Identificación de microorganismos: bacterias y hongos 
sobre una placa de metal pulido re-utilizable. Esta placa permite la aplicación de 96 a 384 muestras para ser analizadas en una misma corrida ${ }^{10}$. Sobre dicha colonia se aplica una matriz en solución (generalmente una solución saturada de ácido alfa-ciano-4-hidroxicinamico, acetonitrilo y ácido trifluoroacético) la que se cristaliza al dejarse secar a temperatura ambiente. Esta matriz cumple dos roles fundamentales; primero expone las proteínas intracelulares mediante la ruptura de la membrana celular y segundo, facilita la vaporización y la ionización de las proteínas mediante un haz de laser pulsante. Estudios iniciales destinados a caracterizar las proteínas evaluadas muestran que éstas son principalmente, pero no exclusivamente, ribosomales ${ }^{14}$. También se encuentran CSP (cold shock protein) y factores de modulación ribosomal ${ }^{11,14}$. Una vez ionizadas estas proteínas viajan por una cámara de vacío siendo detectadas al final. Dependiendo de la relación masa/carga de cada fragmento será el tiempo que ésta demore en llegar al final. Este "tiempo de vuelo" es utilizado para construir el espectro específico de las masas. La manera en que este espectro es analizado va a depender del software utilizado para realizar la identificación ${ }^{12}$. Este espectro es comparado automáticamente con un archivo y el resultado es entregado automáticamente (Figura 1).

En el caso del equipo de Bruker, los resultados son entregados junto a un puntaje. Si éste es igual o mayor a 2 la identificación es posible a nivel de género y especie, entre 1,7 y 1,9 la identificación es sólo a nivel de género y menor a 1,7 no se entrega una identificación. El puntaje requerido para la identificación a nivel de especie pareciera ser bastante conservador. Diversos estudios utilizan puntajes menores para realizar la identificación $(>1,8)^{10,11}$ lo que llevó a De Bel et al. a proponer dos nuevos términos para diferenciar estos criterios: BAC-Bruker Acceptance Criteria (criterio aceptado por Bruker) y LAC-Literature Acceptance Criteria (criterio de aceptación de la literatura $)^{15}$. El uso de criterios aceptados en la literatura científica permitiría la identificación correcta de $26 \%$ más de microorganismos con $2 \%$ de errores a nivel de especie versus $0,7 \%$ de errores de identificación utilizando criterios BAC. Se sugiere por lo tanto, la utilización de otros parámetros asociados para el establecimiento de criterios que podrían permitir aumentar el número de especies correctamente identificadas sin aumentar el número de errores ${ }^{15}$. En el caso de SARAMIS ${ }^{\mathrm{TM}}$ se entrega un valor de confianza asociado al resultado.

Cada vez que se requiere realizar una identificación, debe realizarse una calibración que se lleva a cabo de manera automática utilizando un estándar comercial. Una vez calibrado, la identificación de cada muestra toma uno a dos minutos ${ }^{16}$.

\section{Influencia de los medios de cultivo}

Las proteínas ribosomales, al ser estables y abundantes

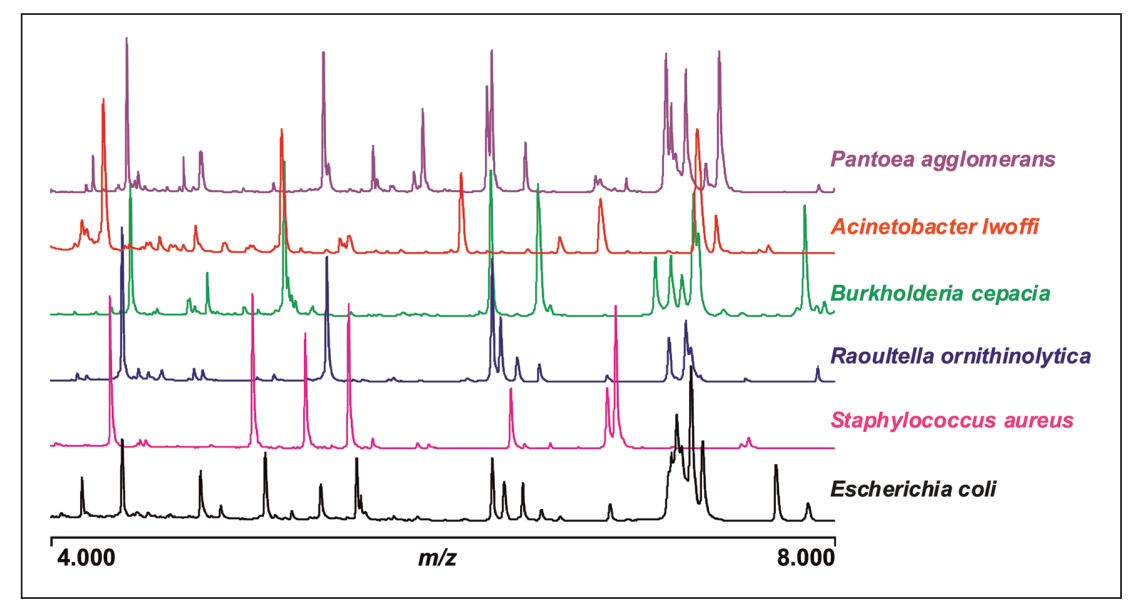

Figura 1. Diferentes perfiles de proteínas obtenidas para diferentes bacterias por MALDI-TOF MS (cortesía bioMerieux).

en su expresión, permitirían en teoría la evaluación por MALDI-TOF MS de distintas cepas, en forma independiente del medio de cultivo o tiempo de incubación. Estudios que evaluaron los espectros obtenidos de distintos medios de cultivo (agar Columbia, Mc Conkey) y a distintas temperaturas muestran una variación que no sería importante a la hora del análisis ${ }^{11}$. En otro estudio se compararon los espectros obtenidos para Escherichia coli, Bacillus subtilis y Yersinia enterocolitica a partir de tripticasa soya, agar sangre y agar Columbia, observándose que no hubo interferencia en la identificación ${ }^{17}$. De esta misma manera, dado que la identificación no se basa en pruebas fenotípicas ni de utilización de sustratos, el tiempo de desarrollo de las colonias no es una limitante en la medida que se observe crecimiento.

En el caso del equipo de Bruker la metodología utilizada para la identificación directa de cultivos sólidos, incluyendo levaduras y micobacterias, está estandarizada por el mismo fabricante. En el caso de identificación directa a partir de los hemocultivos positivos existe disponible un kit comercial para la extracción (MALDI Sepsityper ${ }^{\mathrm{TM}}$ kit).

\section{Identificación de levaduras y micobacterias}

Debido a las características de su pared, ciertos microorganismos como levaduras y micobacterias no pueden ser identificados directamente desde el medio de cultivo, requiriendo de un proceso de extracción previo con la finalidad de exponer las proteínas intracelulares. La extracción consta de centrifugaciones seriadas con etanol, ácido fórmico y acetonitrilo siendo necesario un gabinete de extracción para su realización. El proceso toma seis minutos por muestra pudiendo reducirse si las muestras se trabajan en serie ${ }^{16}$. En el caso de las micobacterias debe realizarse un paso extra en que la muestra debe hervirse a $100^{\circ} \mathrm{C}$ durante cinco minutos para lograr la inactivación de 
las micobacterias y luego agitarse en presencia de perlas de zirconia/silica permitiendo una mayor destrucción de la pared ${ }^{18}$. Protocolos de extracción más sencillos han sido evaluados por otros grupos eliminando el paso de inactivación por calor y agitación con perlas con resultados satisfactorios ${ }^{19,20}$. Una vez finalizada la extracción, se toma una muestra del sobrenadante la que se deposita sobre la placa; una vez seca se procesa de la misma manera que una muestra de colonia directa.

La identificación de micobacterias puede lograrse incluso de medio líquido como el utilizado en los sistemas MGIT aunque el porcentaje de identificación es menor si se compara con colonias tomadas de medio sólido (97 vs $77 \%)^{18}$.

\section{Identificación directa a partir de hemocultivos}

La identificación de microorganismos directamente desde el frasco de hemocultivo ha sido estudiada aplicando procesos de extracción capaces de separar los microorganismos de los otros elementos presentes en la muestra (medio de cultivos, eritrocitos, proteínas plasmáticas). Los procedimientos utilizados en distintos trabajos difieren en algunos aspectos pero requieren tres pasos previos a la identificación: la separación de células, la precipitación de proteínas y la extracción y solubilización de proteínas ${ }^{21}$. Se puede realizar la identificación desde un frasco de hemocultivo inmediatamente después de la alarma de positivización, utilizando desde 0,2 a 5 $\mathrm{mL}$ de sangre. La separación de leucocitos y eritrocitos se realiza por centrifugación; se realiza la lisis celular con agua destilada, cloruro de amonio o saponina, luego se centrifuga, se lava, se precipita con etanol y se realiza una extracción y solubilización con acetonitrilo y ácido fórmico, de manera similar a la realizada en la extracción de levaduras ${ }^{20,22,23}$. Esto permite separar y eliminar los elementos figurados de la sangre, las proteínas plasmáticas y las del medio de cultivo. Se ha descrito que el uso de ácido trifluoroacético en vez de ácido fórmico muestra una menor tasa de identificación ${ }^{22}$.

Uno de los problemas potenciales con respecto a la identificación directa a partir de frascos de hemocultivos es la presencia de más de un microorganismo. Esta situación puede llevar a una no identificación o una identificación errónea. En estos casos es fundamental la observación de la tinción de Gram lo que puede orientar a la presencia de más de un tipo de microorganismo.

\section{Identificación directa a partir de una muestra de orina}

La identificación directa desde muestras de orinas puede realizarse tanto utilizando la orina sin procesar como utilizando protocolos similares a los utilizados en los hemocultivos. De una muestra de orina se toman $4 \mathrm{~mL}$, los que se someten a centrifugaciones sucesivas con velocidades de centrifugación crecientes. El pellet obtenido puede ser aplicado directamente a la placa o ser re-suspendido en etanol, ácido fórmico y acetonitrilo de igual forma que con los hemocultivos. El sobrenadante es luego aplicado a la placa de metal y se trabaja de manera similar a una extracción de levaduras. Ambos métodos permiten la identificación de más de $90 \%$ de las muestras a nivel de género o especie en muestras con más de $10^{5}$ $\mathrm{ufc} / \mathrm{mL}^{24,25}$.

\section{Aplicaciones en microbiología clínica}

Como ya se mencionó, la espectrometría de masas, MALDI-TOF MS en particular, se ha descrito en microbiología desde 1975, pero la mayoría de estas publicaciones fueron realizadas en el área de la microbiología básica y de la espectrometría de masas y proteómica (Tabla 2). No fue hasta el año 2008 en que, en forma simultánea, Mellmann y cols., en Alemania ${ }^{26}$ y Degand y cols., en Francia $^{27}$ utilizando el equipo de Bruker, describen esta metodología en un gran número de bacilos gramnegativos no fermentadores obtenidos de muestras de pacientes. A contar de esa fecha, el incremento de las publicaciones y reportes del uso del MALDI-TOF MS en microbiología clínica ha sido exponencial.

\section{En la identificación de bacterias aerobias, anaerobias, micobacterias y levaduras}

Los resultados de las publicaciones recientes se describen en la Tabla 3, donde se observa que en general el porcentaje de identificaciones correctas por MALDI-TOF MS en comparación con la secuenciación del gen $16 \mathrm{~S}$ ARNr varía entre 93 y 100\%. Es importante destacar que en estas publicaciones los métodos de identificación convencional por métodos bioquímicos tienen una exactitud que oscila entre 83 y $98 \%$ dependiendo del método (API $\AA$, bioMerieux; Vitek $\AA$, bioMerieux; Phoenix $\AA$, Becton Dickinson).

Las diferencias entre los resultados de las publicaciones se pueden explicar por varias razones:

- La capacidad del equipo de asignar un puntaje $\geq 2$ en la identificación de una bacteria y/o levadura a nivel de especie depende de la existencia en la base de datos del fabricante de un determinado espectro de proteínas. A medida que la base de datos aumenta, mejora la capacidad de identificación. En el trabajo de Couturier y cols. ${ }^{28}$, el porcentaje de identificaciones correctas de bacterias del grupo HACEK aumentó a 100\% cuando se utilizó la base de datos del fabricante ampliada con datos del laboratorio, es decir, la aplicabilidad de MALDI-TOF MS es dependiente de las cepas incluidas en la base de datos. Dado lo anterior es importante en la decisión de adquirir un equipo, escoger el que 


\begin{tabular}{|c|c|c|c|}
\hline Año & Autor & Revista & Título \\
\hline 1975 & Anhalt JP & Anal Chem & Identificación de bacterias utilizando espectrometría de masas \\
\hline 1996 & Krishnamurthy $T$ & Rapid Commun Mass Spectrom & Detección de bacterias patogénicas y no patogénicas por espectrometría de masas MALDI-TOF \\
\hline 1996 & Holland RD & Rapid Commun Mass Spectrom & $\begin{array}{l}\text { Identificación rápida de bacterias intactas basada en los patrones espectrales por espectrometría de masas } \\
\text { MALDI-TOF }\end{array}$ \\
\hline 1996 & Claydon MA & Nat Biotechnol & Identificación rápida de bacterias intactas usando espectrometría de masas MALDI-TOF \\
\hline 1999 & Domin MA & Rapid Commun Mass Spectrom & $\begin{array}{l}\text { El efecto de la combinación del solvente y la matriz en el análisis de bacterias por espectrometría de masas } \\
\text { MALDI-TOF }\end{array}$ \\
\hline 2000 & Haag AM & Am Clin Lab. & Rápida identificación de Haemophilus spp y otras bacterias por espectrometría de masas MALDI-TOF \\
\hline 2000 & Jarman KH & Anal Chem & Un algoritmo para la identificación automatizada de bacterias usando espectrometría de masas MALDI-TOF \\
\hline 2001 & Lay JO Jr. & Mass Spectrom Rev & Espectrometría de masas MALDI-TOF de bacterias \\
\hline 2001 & Fenselau C & Mass Spectrom Rev & Caracterización por microorganismos intactos por espectrometría de masas MALDI-TOF \\
\hline 2002 & Bright JJ & J Microbiol Methods & Rápida tipificación de bacterias usando MALDI-TOF y un software de reconocimiento \\
\hline 2002 & Bernardo KN & Proteomics & Identificación y discriminación de Staphylococcus aureus usando espectrometría de masas MALDI-TOF \\
\hline 2005 & Dworzanski JP & Expert Rev Proteomics & Clasificación e identificación de bacterias usando espectrometría de masas basada en proteómica \\
\hline 2005 & Valentine $\mathrm{N}$ & Applied Environ Microbiol & $\begin{array}{l}\text { Efecto de las condiciones del cultivo en la identificación de microorganismos por espectrometría de masas } \\
\text { MALDI-TOF }\end{array}$ \\
\hline 2008 & Mellmann A & J ClinMicrobiol & $\begin{array}{l}\text { Evaluación de espectrometría de masas MALDI-TOF en comparación con la secuenciación del gen } 16 \mathrm{~S} \text { ARNr } \\
\text { para la identificación de especies en bacilos gramnegativos no fermentadores }\end{array}$ \\
\hline 2008 & Degand N & J ClinMicrobiol & $\begin{array}{l}\text { Espectrometría de masas MALDI-TOF para la identificación de bacilos gramnegativos no fermentadores } \\
\text { aislados de pacientes con fibrosis quística }\end{array}$ \\
\hline
\end{tabular}

tenga la base de datos más extensa, el que permita la incorporación de nuevos perfiles proteicos de bacterias correctamente identificadas por el método de referencia $\mathrm{y}$ aquel en que el fabricante actualice frecuentemente la base de datos.

- Un paso de extracción manual previo a la carga de la cepa en los pocillos mejora el puntaje, ya que permite una mejor exposición de las proteínas al láser.

- La identificación de algunas bacterias es limitada pues su perfil proteico es similar a otras y porque al momento de ser evaluadas existían pocos datos en la base del fabricante, por ejemplo Streptococcus pneumoniae con Streptococcus del grupo viridans, Shigella spp, Klebsiella oxytoca.

MALDI-TOF MS permite muy buenas identificaciones en Enterobacteriaceae $e^{10,29-35}$, con excepción de Shigella que es identificada erróneamente como $E$. coli y no está incluida en la base de datos porque tienen perfiles de proteínas muy similares por estar filogenéticamente relacionadas. En el caso del género Salmonella, es posible la diferenciación de serovars de Salmonella enterica subespecie enterica especialmente serovars Enteritidis, Typhimurium, Virchow, Infantis y $\mathrm{Hadar}^{36}$. Se han descrito dificultades en la identificación con puntaje $<2$ en Enterobacter cloacae complex ${ }^{37}$, un grupo de seis especies que son íntimamente relacionadas (E. cloacae, E. asburiae, E. hormaechi, E. kobei, E. ludwigii y E. nimipressuralis), por lo cual su diferenciación no es posible todavía.

En el caso de los bacilos gramnegativos no fermentadores ${ }^{10,29-35,38}$, donde los sistemas convencionales son poco exactos para aquellas especies más infrecuentes, MALDI-TOF MS ha demostrado mucha utilidad. Para Stenotrophomas maltophilia, los primeros estudios fueron publicados cuando su perfil proteico no estaba incluido en la base de datos ${ }^{10,30,31}$. En la identificación de bacterias del grupo HACEK ${ }^{28,35}$ y otras fastidiosas, en bacilos gramnegativos curvos del grupo de Campylobacter, Arcobacter y Helicobacter ${ }^{29,33,35,39}$ y Legionella ${ }^{40}$, las identificaciones correctas superan el 94\%.

En cuanto a la identificación de especies grampositivas, MALDI-TOF MS presenta un excelente rendimiento en Staphylococcus $\mathrm{sp}^{10,29-32}$ considerándose un excelente método, costo-efectivo y rápido para la identificación de especie en Staphylococcus coagulasa negativa, donde presenta 93\% de identificaciones correctas en relación a $75 \%$ de identificaciones correctas con Phoenix ${ }^{\circledR}$, Becton Dickinson y Vitek-2®, bioMerieux, respectivamente. Para lo anterior se utilizó como estándar de oro la secuenciación del gen $\operatorname{sod} A$ en 243 muestras que representaban 20 especies $^{41}$. También se han obtenido buenos resultados 
Tabla 3. Rendimiento de la espectrometría de masas MALDI-TOF en microbiología clínica en la identificación (ID) de bacterias aeróbicas y anaeróbicas en comparación con métodos convencionales ${ }^{\&}$

\begin{tabular}{|c|c|c|c|c|c|}
\hline $\begin{array}{l}\text { Año y } \\
\text { autor }\end{array}$ & $\begin{array}{l}\text { Grupos bacterianos es- } \\
\text { tudiados }\end{array}$ & $\begin{array}{c}n \\
\text { total }\end{array}$ & $\begin{array}{l}\text { \% ID correctas } \\
\text { MALDI-TOF* }\end{array}$ & $\begin{array}{l}\text { ID con problemas } \\
\text { MALDI-TOF (\% error) }\end{array}$ & Observaciones \\
\hline $\begin{array}{l}2009 \\
\text { Seng } 10\end{array}$ & $\begin{array}{l}\text { Aerobios } \\
\text { Anaerobios }\end{array}$ & 1.660 & $95,4 \%$ & $\begin{array}{l}\text { Anaerobios (30) } \\
\text { Streptococcus pneumoniae (46) } \\
\text { Shigella sonnei (100) } \\
\text { Stenotrophomonas maltophilia (70) } \\
\text { Enterobacter spp (4) }\end{array}$ & $\begin{array}{l}\text { Los métodos convencionales identificaron correctamente } \\
\text { el } 98 \% \text { de los aislados } \\
\text { MALDI-TOF identificó a nivel de especie } 84,1 \% \text { de los } \\
\text { aislados } \\
\text { Las fallas en la ID se deben principalmente a que los espec- } \\
\text { tros no existían en la base de datos }\end{array}$ \\
\hline $\begin{array}{l}2009 \\
\text { Lartigue }^{42}\end{array}$ & Streptococcus agalactiae & 110 & $100 \%$ & - & MALDI-TOF permite establecer líneas filogenéticas \\
\hline $\begin{array}{l}2010 \\
\text { van Veen } 29\end{array}$ & $\begin{array}{l}\text { Aerobios, anaerobios } \\
\text { Levaduras }\end{array}$ & 327 & $95,1 \%$ & $\begin{array}{l}\text { S. pneumoniae (13) } \\
\text { Streptococcus grupo viridans ( } 57)\end{array}$ & Método convencional identificó correctamente sólo 92,2\% \\
\hline $\begin{array}{l}2010 \\
\text { Ferreira }\end{array}$ & $\begin{array}{l}\text { Aerobios } \\
\text { Anaerobios }\end{array}$ & 294 & $\begin{array}{l}100 \% \text { en } \operatorname{Gram}(+) \\
97,7 \% \text { en } \operatorname{Gram}(-)\end{array}$ & $\begin{array}{l}\text { Raoultella sp } \\
\text { Acinetobacter spp } \\
\text { S. maltophilia } \\
\text { Francisella }\end{array}$ & $\begin{array}{l}\text { MALDI-TOF identificó correctamente a nivel de especie } \\
87,7 \% \text { de bacilos gramnegativos }\end{array}$ \\
\hline $\begin{array}{l}2010 \\
\text { Bizzini }^{31}\end{array}$ & $\begin{array}{l}\text { Gram }(+) \text { aerobios } \\
\text { Gram }(-) \text { aerobios } \\
\text { Levaduras }\end{array}$ & 1.371 & $98,7 \%$ & $\begin{array}{l}\text { S. sonnei } \\
\text { Propionibacterium acnes } \\
\text { S. maltophilia } \\
\text { Agrobacterium sp } \\
\text { Enterobacter spp } \\
\text { Haemophilus parainfluenzae }\end{array}$ & $\begin{array}{l}\text { MALDI-TOF identificó correctamente a nivel de especie } \\
93,2 \% \text { de los aislamientos } \\
\text { Las fallas en la ID se deben principalmente a que los espec- } \\
\text { tros no existían en la base de datos } \\
\text { Un paso de extracción adicional mejora el porcentaje de } \\
\text { identificación }\end{array}$ \\
\hline $\begin{array}{l}2010 \\
\text { Stephan }\end{array}$ & Cronobacter spp & 36 & $100 \%$ & - & $\begin{array}{l}\text { Cronobacter sp es patógeno oportunista asociado a infec- } \\
\text { ción neonatal }\end{array}$ \\
\hline $\begin{array}{l}2010 \\
\text { Bessede }^{32}\end{array}$ & $\begin{array}{l}\text { Gram }(+) \text { aerobios } \\
\text { Gram }(-) \text { aerobios } \\
\text { Anaerobios }\end{array}$ & 1.013 & $97,3 \%$ & $\begin{array}{l}\text { Chryseobacterium sp } \\
\text { P. acnes } \\
\text { Citrobacter sp } \\
\text { Lactobacillus sp }\end{array}$ & $\begin{array}{l}\text { Un paso de extracción cuando score }<2 \text { mejora el por- } \\
\text { centaje de identificación. Método convencional identificó } \\
\text { correctamente sólo el } 93,2 \%\end{array}$ \\
\hline $\begin{array}{l}2010 \\
\mathrm{He}^{33}\end{array}$ & $\begin{array}{l}\text { Bacilos entéricos } \\
\text { (Escherichia coli, Salmonella } \\
\text { sp, Shigella, Yersinia sp, } \\
\text { Campylobacter sp) }\end{array}$ & 304 & 68 & $\begin{array}{l}\text { Shigella sonnei } \\
\text { Shigella spp } \\
\text { Escherichia coli } \\
\text { enterohemorrágica }\end{array}$ & $\begin{array}{l}\text { Shigella sonnei no está en la base de datos, la identifica } \\
\text { como } E \text {. coli }\end{array}$ \\
\hline $\begin{array}{l}2011 \\
\text { Benagli34 }\end{array}$ & $\begin{array}{l}\text { Gram }(+) \text { aerobios } \\
\text { Gram }(-) \text { aerobios }\end{array}$ & 1.019 & $94,7 \%$ & $\begin{array}{l}\text { Enterobacter } \\
\text { Klebsiella oxytoca } \\
\text { S. pneumoniae }\end{array}$ & $\begin{array}{l}\text { Un } 63 \% \text { de las ID por MALDI-TOF fueron confirmados } \\
\text { por la secuenciación del gen } 16 \text { SARNr vs } 11 \% \text { de las ID } \\
\text { por VITEK }\end{array}$ \\
\hline $\begin{array}{l}2011 \\
\text { Couturier }^{28}\end{array}$ & Grupo HACEK & 103 & $93 \%$ & - & $\begin{array}{l}\text { Cuando se usa una base de datos ampliada (Bruker+ } \\
\text { propia del laboratorio) las ID correctas aumentan a 100\% } \\
\text { comparados con secuenciación del gen 16SrRNA }\end{array}$ \\
\hline $\begin{array}{l}2011 \\
\text { Saffert }\end{array}$ & Bacilos Gram (-) & 440 & $93 \%$ & $\begin{array}{l}\text { Raoultella spp } \\
\text { Enterobacter spp } \\
\text { K. oxytoca } \\
\text { Shigella spp }\end{array}$ & $\begin{array}{l}\text { MALDI-TOF identifica significativamente mejor que BD } \\
\text { Phoenix (93 vs 83\%) }\end{array}$ \\
\hline
\end{tabular}

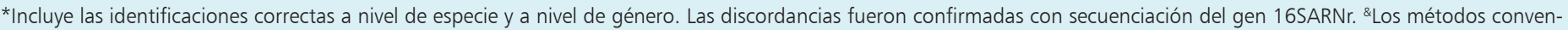
cionales fueron Vitek, API, BD Phoenix.

en Streptococcus $\operatorname{sp}^{10,29,30,32,34,42}$ con excepción de $S$. pneumoniae y Streptococcus parasanguinis del grupo mitis ya que tienen perfiles proteicos muy parecidos, por lo que el fabricante recomienda una prueba fenotípica adicional para su identificación; lo que no representa un problema de error de identificación ya que el puntaje es generalmente bajo. En el género Enterococcus la diferenciación de E. faecium y E. fecalis ha mostrado una alta exactitud y representa una ayuda en la diferen- ciación entre E. faecium y E. gallinarum y E. flavus, que por métodos convencionales en muy inexacta ${ }^{29,31,32}$. Del mismo modo, resulta muy útil en cocáceas grampositivas catalasa negativa como Abiotrophia sp, Granulicatella sp, Globicatella sp, Vagococcus sp, Aerococcus sp y Gemella $\mathrm{sp}^{29,32}$, cuya identificación por métodos convencionales es lenta y engorrosa, ya que los sistemas automatizados no alcanzan buena exactitud.

En anaerobios, MALDI-TOF MS ha mostrado una 
excelente correlación con la secuenciación del gen $16 \mathrm{~S}$ ARNr especialmente en Clostridium sp y Bacteroides $\mathrm{sp}$ principalmente en B. thetaiotaomicron, B. ovatus y $B$. uniformis. En Propionibacterium acnes los problemas de identificación iniciales se debieron a la ausencia de referencia en la base de datos de Bruker ${ }^{10,31,32}$. En el caso de Fusobacterium nucleatum, Prevotella sp y Peptostreptococcus sp, es necesario ampliar la base de datos para la obtención de identificaciones a nivel de especie con puntaje $\geq 2$. Cuando los espectros de anaerobios están en la base de datos, la identificación por espectrometría de masas es mejor que la identificación convencional ${ }^{10,31,32,43}$.

En levaduras, la identificación por MALDI-TOF MS en los trabajos iniciales fue de $85 \%$ en 61 cepas de 12 especies diferentes ${ }^{29,31}$. Este porcentaje ha mejorado con la ampliación de la base de datos y con un proceso de extracción previo al análisis, lo que permite obtener mejores espectros. Marklein y cols., estudiaron 267 aislados de 18 especies, obteniendo concordancia con los métodos fenotípicos en 92,5\% (247/267). Con la ampliación de la base de datos mediante secuenciación del gen 26S ARNr, se logró una identificación correcta de $100 \%$ de las levaduras estudiadas que incluían los géneros Candida, Cryptococcus, Saccharomyces. Trycosporon, Geotrichum, Pichia y Blastoschizomyces ${ }^{44}$.

\section{En la identificación de bacterias y levaduras} directamente desde hemocultivos y urocultivos

La identificación correcta de microorganismos directamente a partir de muestras de orina depende de la carga bacteriana de la muestra. Ferreira y cols., estudiaron 218 muestras positivas por urocultivo, encontrando que cuando la muestra tenía $>100.000$ ufc/mL la correlación con el urocultivo fue de $91,8 \%$ a nivel de especie y de $92,7 \%$ a nivel de género ${ }^{24}$. Cuando los urocultivos fueron negativos la correlación fue de $100 \%{ }^{25}$. Los autores concluyen que MALDI-TOF MS es un método rápido, con alta exactitud, especialmente cuando las muestras poseen una alta carga bacteriana, en particular de bacilos gramnegativos.

Para la identificación directa a partir de hemocultivos positivos en sistemas automatizados hay varias referencias que muestran la utilidad de utilizar MALDI-TOF $\mathrm{MS}^{6,24,45-49}$. Como se explicó anteriormente, para obtener espectros a partir de los hemocultivos, es necesario un proceso previo de extracción de los componentes de la sangre y del medio de cultivo. En general, los resultados son mejores cuando los recuentos bacterianos son altos, cuando las bacteriemias son monobacterianas y cuando los agentes son bacilos gramnegativos, obteniendo resultados concordantes con los sub-cultivos en aproximadamente $95 \%$ a nivel de género y $83 \%$ a nivel de especie. En septiembre de 2010, Bruker comercializó un kit que permite la extracción más rápida a partir de la muestra obtenida de las botellas de hemocultivos, con lo cual el tiempo de procesamiento se acorta y simplifica bastante (MALDI Sepsityper ${ }^{\mathrm{TM}}$ kit). Esta metodología se considera RUO (Research Use Only) para fuera de la Unión Europea y en Europa es considerada IVD-CE (In vitro Device). Para la identificación directa de fungiemias por levaduras, MALDI-TOF MS ha mostrado una correlación de género y especie de $91 \%$, con resultados obtenidos en 20 minutos $^{47}$. En la Tabla 4 se muestra un análisis comparativo de la utilidad de MALDI-TOF MS en muestras directas del hemocultivos con otras metodologías para pacientes bacteriémicos ${ }^{6}$.

\section{Limitaciones}

Las limitaciones en identificación bacteriana entregadas por Bruker Daltonics como fabricante se describen en la Tabla 5 y han sido comentadas previamente. La ampliación de las bases de datos por los usuarios mejorará mucho la capacidad de identificación al superar los actua-

\begin{tabular}{|c|c|c|c|c|c|c|c|}
\hline $\begin{array}{l}\text { Método para ID* bacteriana en } \\
\text { bacteriemia y sepsis }\end{array}$ & $\begin{array}{l}\text { Experiencia técnica } \\
\text { requerida }\end{array}$ & $\begin{array}{l}\text { Carga } \\
\text { trabajo }\end{array}$ & $\begin{array}{l}\text { Costo de } \\
\text { reactivos }\end{array}$ & $\begin{array}{l}\text { Estandarización/ } \\
\text { Reproducibilidad }\end{array}$ & $\begin{array}{c}\% \text { ID } \\
\text { especie }\end{array}$ & $\begin{array}{c}\% \text { ID } \\
\text { correcta }\end{array}$ & Impacto clínico \\
\hline $\begin{array}{l}\text { Hemocultivo + Gram + subcultivo+ } \\
\text { ID bioquímica }\end{array}$ & Alta & Alta & Moderado & Método estándar & 97 & 98 & Método estándar \\
\hline $\begin{array}{l}\text { Hemocultivo +Gram + subcultivo+ } \\
\text { ID bioquímica +MALDI-TOF }\end{array}$ & Baja & Baja & Bajo & $\begin{array}{l}\text { Alta/Alta } \\
\text { En laboratorios con } \\
\text { experiencia }\end{array}$ & 97 & 98 & Más rápido \\
\hline Hemocultivo + MALDI-TOF & Baja & Media & Bajo & $\begin{array}{l}6 \text { artículos } \\
\text { Kit comercial } \\
\text { SepsiTyper }\end{array}$ & - & - & Más rápido \\
\hline Hemocultivo + RPC + microarreglo & Alta & Alta & No descrito & $\begin{array}{l}\text { Kit comerciales } \\
\text { Muy pocos artículos }\end{array}$ & No descrito & No descrito & No descrito \\
\hline Septi-Fast & Muy alta & Muy alta & Muy alto & $\begin{array}{l}\text { Kit comerciales } \\
\text { Muchos artículos }\end{array}$ & - & - & Indicador de gravedad \\
\hline
\end{tabular}


Tabla 5. Limitaciones en la identificación por MALDI Biotyper®, Bruker

\begin{tabular}{|c|c|}
\hline Género o grupo bacteriano & Limitación \\
\hline Shigella spp & $\begin{array}{l}\text { No está incluido en la base de datos, por encontrarse filogenéticamente relacionado con Escherichia coli, con patrones } \\
\text { proteicos muy similares }\end{array}$ \\
\hline Streptococcus pneumoniae & Requiere conformación por un test adicional, por su cercanía filogenética con el grupo mitis \\
\hline Stenotrophomonas maltophilia & $\begin{array}{l}\text { Muy relacionada con Pseudomonas hibiscola, P. geniculata y P. beteli, por lo que las identifica como Grupo Steno- } \\
\text { trophomonas maltophilia }\end{array}$ \\
\hline Acinetobacter baumannii-calcoaceticus complex & No se puede diferenciar entre Acinetobacter baummannii, A. calcoaceticus, A. genoespecie 3, A. genoespecie 13 \\
\hline Enterobacter cloacae complex & $\begin{array}{l}\text { No se puede diferenciar entre } 6 \text { especie muy relacionadas E. cloacae, E. asburiae, E. hormaechi, E. kobei, E. ludwigii } \\
\text { y E. nimipressuralis }\end{array}$ \\
\hline Burkholderia cepacia complex & No diferencia cada miembro del grupo \\
\hline Bordetella pertussis - Bordetella bronchiseptica & Patrón muy similar \\
\hline Achromobacter xylosoxidans - Achromobacter ruhlandii & Patrón muy similar \\
\hline Grupo Pseudomonas putida & No diferencia cada miembro del grupo \\
\hline Grupo Pseudomonas fluorescens & No diferencia cada miembro del grupo \\
\hline Bacteroides nordii - Bacteroides salyersiae & Patrón muy similar \\
\hline Candida africana & No diferencia este subgrupo dentro de Candida albicans \\
\hline Klebsiella oxytoca - Raoultella ornitholytica & Patrón muy similar \\
\hline Género Aeromonas & No diferencia algunas especies dentro del género \\
\hline
\end{tabular}

les bajos puntajes. Por otra parte, la demora y laboriosidad del manejo de microorganismos que requieren procesos previos de extracción también se irán simplificando con la comercialización de kits de extracción. Probablemente un problema que no podrá ser resuelto cuando se utilice esta metodología directa de las muestras es la dificultad en la identificación de mezclas de microorganismos.

Otra limitación actual del proceso es que la inoculación de las cepas es manual, por lo que siempre existe el riesgo de error en la posición en que se cargan las muestras.

Respecto al costo del equipo, una evaluación económica realizada en nuestro laboratorio permite estimar que la inversión se recupera en cuatro años, considerando el ahorro en insumos de laboratorio, tiempo en mano de obra y en repeticiones de la identificación microbiológica por los métodos manuales convencionales.

\section{Conclusión}

El uso de la tecnología de espectrometría de masas, específicamente MALDI-TOF MS, en la identificación microbiológica en los laboratorios clínicos es, sin duda, uno de los cambios más importantes después de la introducción de los métodos moleculares.

$\mathrm{Su}$ aplicación en la identificación bacteriana y de levaduras en un laboratorio clínico, permite acortar en forma significativa los tiempos de respuesta, mejorar la exactitud de los métodos de identificación convencional y disminuir los altos costos involucrados, tanto en uso de reactivos e insumos como en mano de obra. Estimaciones indican que el costo de la identificación oscila entre US $\$ 0,5$ y US $\$ 3$ y que el tiempo promedio requerido para la identificación es de seis minutos ${ }^{10}$. Un análisis de costos realizado en nuestro laboratorio mostró que el precio por cada determinación en duplicado, considerando todos los materiales y el tiempo de procesamiento por un profesional, es de US $\$ 1,6$.

Hacia el futuro se puede vislumbrar que esta tecnología podrá ser utilizada en la detección de determinantes de resistencia bacteriana y en la detección de factores de patogenicidad. Cuando se logren las validaciones para estas aplicaciones, sin duda MALDI-TOF MS será la metodología más utilizada en nuestros laboratorios.

A todo lo anterior se agrega el uso de la espectrometría de masas como estándar de oro en las distintas áreas del laboratorio clínico y la posibilidad de uso de MALDI-TOF MS en aplicaciones clínicas de proteómica y metabolómica.

\section{Resumen}

La identificación bacteriana es muy importante en el manejo adecuado de los pacientes infectados, especialmente aquellos con infecciones graves hospitalizados en unidades de pacientes críticos. La identificación por los métodos convencionales utilizados en los laboratorios de microbiología clínica demora al menos 16 horas desde 
que un cultivo es positivo. La introducción de la espectrometría de masas, específicamente del espectrómetro de masas por tiempo de migración (tiempo de vuelo) con desorción/ionización laser asistida por una matriz (MALDI-TOF MS, por su sigla en inglés matrix-assisted laser desorption/ionization time-of-flight mass spectrometer), en el laboratorio de microbiología podría significar un cambio radical en la precisión de la identificación, el tiempo de detección (6 minutos por bacterias) y el costo (aproximadamente 5 veces más económico que la identificación convencional). Desde su introducción en los laboratorios de microbiología clínica en el año 2008, se han escrito numerosas publicaciones sobre su utilidad en la identificación de microorganismos desde colonias, así como directamente desde hemocultivos positivos y de muestras de orina. Esta revisión describe la metodología de MALDI-TOF MS, su rendimiento en la identificación de bacterias aerobias, anaerobias, micobacterias y levaduras, sus futuras aplicaciones en microbiología y sus principales desventajas.

\section{Referencias bibliográficas}

1.- Carroll K C, Weinstein M P. Manual and automated systems for detection and identification of microorganisms. In: Murray P R, Baron E J, Jorgensen J H, Landry M L, Pfaller M A, eds. Manual of Clinical Microbiology. 9th ed. Washington, DC American Society for Microbiology 2007; 192-217.

2.- Harbarth S, Garbino J, Pugin J, Romand J A, Lew D, Pittet D. Inappropriate initial antimicrobial therapy and its effect on survival in a clinical trial of immunomodulating therapy for severe sepsis. Am J Med 2003; 115: 529-35.

3.- Ibrahim E H, Sherman G, Ward S, Fraser V J, Kollef M H. The influence of inadequate antimicrobial treatment of bloodstream infections on patient outcomes in the ICU setting. Chest 2000; 118: 146-55.

4.- Kumar A, Ellis P, Arabi Y, Roberts D, Light B, Parrillo J E, et al. Initiation of inappropriate antimicrobial therapy results in a fivefold reduction of survival in human septic shock. Chest 2009; 136: 1237-48.

5.- Kumar A, Roberts D, Wood K E, Light B, Parrillo J E, Sharma S, et al. Duration of hypotension before initiation effective antimicrobial therapy the critical determinant of survival in human septic shock. Crit Care Med 2006; 34: 1589-96

6.- Leggieri N, Rida A, Francois P, Schrenzel J. Molecular diagnosis of bloodstream infections: planning to (physically) reach the bedside. Curr Opin Infect Dis 2010; 23: 311-9.

7.- Holmes B, Willcox W R, Lapage S P. Identification of Enterobacteriaceae by the API 20E system. J Clin Pathol 1978; 31: 22-30.

8.- Evangelista A T, Truant A L, Bourbeau P P. Chapter 3: Rapid systems and instruments for the identification of bacteria. Truant A L. Manual of Commercial Methods in Clinical Microbiology. Washington DC: ASM Press, 2002, p. 22-9.

9.- $\quad$ Anhalt J P, Fenselau C. Identification of bacteria using mass spectrometry. Anal Chem 1975; 47 : 219-25.

10.- Seng P, Drancourt M, Gouriet F, La Scola
B, Fournier PE, Rolain J M, et al. Ongoing revolution in bacteriology: Routine identification of bacteria by matrix-assisted laser desorption ionization time-of-flight mass spectrometry. Clin Infect Dis 2009; 49: 543-51.

11.- Welker M, Moore E R. Applications of whole cell matrix assisted laser desorption/ionization time of flight mass spectrometry in systematic microbiology. Syst Appl Microbiol 2011; 34: 2-11.

12.- Emonet S, Shah H N, Cherkaoui A, Schendrel J. Application and use of various mass spectrometry methods in clinical microbiology. Clin Microbiol Infect 2010; 16: 1604-13.

13.- Sauer S, Kliem M. Mass spectrometry tools for the classification and identification of bacteria. Nat Rev Microbiol 2010; 8 (1): 74-82.

14.- Ryzhow V, Fenselau C. Characterization of the protein subset desorbed by MALDI from whole bacterial cells. Anal Chem 2001; 4: 746-50.

15.- De Bel A, Wybo I, Vandoorslaer K, Rosseel P, Lauwers S, Piérard D. Acceptance criteria for identification results of Gram-negative rods by mass spectrometry. J Med Microbiol 2011; 60: 684-6.

16.- Bizzini A, Greub G. Matrix-assisted laser desoption ionization time of flight mass spectrometry, a revolution in clinical microbial identification. Clin Microbiol Infect 2010; 16: 1614-9.

17.- Valentine N, Wunschel S, Wunschel D, Petersen C, Wahl K. Effect of culture conditions on microorganism identification by matrixassisted laser desorption ionization mass spectrometry. Appl Environ Microbiol 2005; 71: 58-64.

18.- Manual del Usuario para MALDI-TOF en Autoflex MS. Bruker Daltonics.

19.- Lotz A, Ferroni A, Beretti J L, Dauphin B, Carbonnelle E, Guet-Revillet H, et al. Rapid identification of mycobacterial whole cell in solid and liquid culture media by matrixassisted laser desorption ionization time-offlight mass spectrometry. J Clin Microbiol 2010; 48: 4481-6.

20.- Saleeb P, Drake S K, Murray P, Zelazny A M. Identification of mycobacteria in solid culture media by matrix-assisted laser desorption ionization time-of-flight mass spectrometry. J Clin Microbiol 2011; 49: 1970-794.

21.- Drancourt M. Detection of microorganisms in blood specimens using matrix-assisted laser desorption ionization time-of-flight mass spectrometry: a review. Clin Microbiol Infect 2010; 16: 1620-5.

22.- Ferreira L, Vega Castaño S, Sánchez-Juanes F, González-Cabrero S, Menegotto F, OrduñaDomingo A, et al. Identification of Brucella by MALDI-TOF mass spectrometry. Fast and reliable identification from agar plates and blood cultures. PLoS One 2010; 5: e14235.

23.- La Scola B, Raoult D. Direct identification of bacteria in positive blood culture bottles by matrix-assisted laser desorption ionization timeof-flight mass spectrometry. PLoS one 2009; 4: e8041.

24.- Ferreira L, Sánchez-Juanes F, Muñoz Bellido J L, González-Buitrago J M. Rapid method for direct identification of bacteria in urine and blood culture samples by matrix-assisted laser desorption ionization time-of-flight mass spectrometry: intact cell vs. extraction method. Clin Microbiol Infect 2011; 17 (7): 1007-12.

25.- Ferreira L, Sánchez-Juanes F, González-Ávila M, Cembrero-Fuciños D, Herrero-Hernández A, González-Buitrago JM. Direct identification of urinary tract pathogens from urine samples by MALDI TOF (matrix-assisted laser desorption ionization time-of-flight mass spectrometry). J Clin Microbiol 2010; 48: 2110-5.

26.- Mellmann A, Cloud J, Maier T, Keckevoet U, Ramminger I, Iwen P, et al. Evaluation of matrix-assisted laser desorption ionizationtime-of-flight mass spectrometry in comparison to $16 \mathrm{~S}$ rRNA gene sequencing for species identification of non fermenting bacteria. J Clin Microbiol 2008; 46: 1946-54.

27.- Degand N, Carbonnelle E, Dauphin B, Beretti J L, Le Bourgeois M, Sermet-Gaudelus I, et al. Matrix-assisted laser desorption ionization-time of flight mass spectrometry for identification of non fermenting gram-negative bacilli isolated from cystic fibrosis patients. J Clin Microbiol 2008; 46: 3361-7.

28.- Couturier M R, Mehinovic E, Croft A C, Fisher M A. Identification of HACEK clinical 
isolates by matrix-assisted laser desorption ionization-time of flight mass spectrometry. J Clin Microbiol 2011; 49: 1104-6.

29.- van Veen S Q, Claas E C, Kuijper E J. Highthroughput identification of bacteria and yeast by matrix-assisted laser desorption ionizationtime of flight mass spectrometry in conventional medical microbiology laboratories. J Clin Microbiol 2010; 48: 900-7.

30.- Ferreira L, Vega S, Sánchez-Juanes F, González M, Herrero A, Muñiz MC, et al. Identifying bacteria using a matrix-assisted laser desorption ionization time-of-flight (MALDITOF) mass spectrometer. Comparison with routine methods used in clinical microbiology laboratories. Enferm Infecc Microbiol Clin 2010; 28: 492-7.

31.- Bizzini A, Durussel C, Bille J, Greub G, Prod'hom G. Performance of matrix-assisted laser desorption ionization-time of flight mass spectrometry for identification of bacterial strains routinely isolated in a clinical microbiology laboratory. J Clin Microbiol 2010; 48: 1549-54.

32.- Bessède E, Angla-Gre M, Delagarde Y, Sep Hieng S, Ménard A, Mégraud F. Matrix-assisted laser-desorption/ionization biotyper: experience in the routine of a University hospital. Clin Microbiol Infect 2011; 17: 533-8.

33.- He Y, Li H, Lu X, Stratton CW, Tang YW. Mass spectrometry biotyper system identifies enteric bacterial pathogens directly from colonies grown on selective stool culture media. J Clin Microbiol 2010; 48: 3888-92.

34.- Benagli C, Rossi V, Dolina M, Tonolla M, Petrini O. Matrix-assisted laser desorption ionization-time of flight mass spectrometry for the identification of clinically relevant bacteria. PLoS One 2011; 6: e16424.

35.- Saffert R T, Cunningham S A, Ihde S M, Jobe K E, Mandrekar J, Patel R. Comparison of Bruker Biotyper matrix-assisted laser desorption ionization-time of flight mass spectrometer to BD Phoenix automated microbiology system for identification of gramnegative bacilli. J Clin Microbiol 2011; 49: $887-92$.
36.- Dieckmann R, Malorny B. Rapid screening of epidemiologically important Salmonella enterica subsp. enterica serovars by whole-cell matrix-assisted laser desorption ionizationtime of flight mass spectrometry. Appl Environ Microbiol 2011; 77: 4136-46.

37.- Hoffmann H, Stindl S, Ludwig W, Stumpf A, Mehlen A, Monget D, et al. Enterobacter hormaechei subsp. oharae subsp. nov., $E$. hormaechei subsp. hormaechei comb. nov., and E. hormaechei subsp. steigerwaltii subsp. nov., three new subspecies of clinical importance. J Clin Microbiol 2005; 43 (7): 3297-303.

38.- Mellmann A, Bimet F, Bizet C, Borovskaya A D, Drake R R, Eigner U, et al. High interlaboratory reproducibility of matrix-assisted laser desorption ionization-time of flight mass spectrometry-based species identification of non fermenting bacteria. J Clin Microbiol 2009; 47: 3732-4.

39.- Alispahic M, Hummel K, Jandreski-Cvetkovic D, Nöbauer K, Razzazi-Fazeli E, Hess M, et al. Species-specific identification and differentiation of Arcobacter, Helicobacter and Campylobacter by full-spectral matrixassociated laser desorption/ionization time of flight mass spectrometry analysis. J Med Microbiol 2010; 59: 295-301.

40.- Moliner C, Ginevra C, Jarraud S, Flaudrops C, Bedotto M, Couderc C, et al. Rapid identification of Legionella species by mass spectrometry. J Med Microbiol 2010; 59: 27384.

41.- Dupont C, Sivadon-Tardy V, Bille E, Dauphin B, Beretti JL, Álvarez A S, et al. Identification of clinical coagulase-negative staphylococci, isolated in microbiology laboratories, by matrix-assisted laser desorption/ionization-time of flight mass spectrometry and two automated systems. Clin Microbiol Infect 2010; 16: 9981004.

42.- Lartigue M F, Héry-Arnaud G, Haguenoer E, Domelier A S, Schmit P O, van der MeeMarquet N, et al. Identification of Streptococcus agalactiae isolates from various phylogenetic lineages by matrix-assisted laser desorption ionization-time of flight mass spectrometry.
J Clin Microbiol 2009; 47: 2284-7.

43.- Nagy E, Maier T, Urban E, Terhes G, Kostrzewa M. ESCMID Study Group on Antimicrobial Resistance in Anaerobic Bacteria. Species identification of clinical isolates of Bacteroides by matrix-assisted laser-desorption/ ionization time-of-flight mass spectrometry. Clin Microbiol Infect 2009; 15: 796-802.

44.- Marklein G, Josten M, Klanke U, Müller E, Horré R, Maier T, et al. Matrix-assisted laser desorption ionization-time of flight mass spectrometry for fast and reliable identification of clinical yeast isolates. J Clin Microbiol 2009; 47: 2912-7.

45.- Stevenson L G, Drake S K, Murray P R. Rapid identification of bacteria in positive blood culture broths by matrix-assisted laser desorption ionization-time of flight mass spectrometry. J Clin Microbiol 2010; 48: 444-7.

46.- Ferreira L, Sánchez-Juanes F, Porras-Guerra I, García-García M I, García-Sánchez J E, González-Buitrago J M, et al. Microorganisms direct identification from blood culture by matrix-assisted laser desorption/ionization timeof-flight mass spectrometry. Clin Microbiol Infect 2011; 17: 546-51.

47.- Ferroni A, Suárez S, Beretti J L, Dauphin B, Bille E, Meyer J, et al. Real-time identification of bacteria and Candida species in positive blood culture broths by matrix-assisted laser desorption ionization-time of flight mass spectrometry. J Clin Microbiol 2010; 48 : 1542-8.

48.- Yan Y, He Y, Maier T, Quinn C, Shi G, Li H, et al. Improved Identification of yeast species directly from positive blood culture media by combining Sepsityper specimen processing and Microflex analysis with the MALDI biotyper system. J Clin Microbiol 2011; 49 (7): 2528-32.

49.- Christner M, Rohde H, Wolters M, Sobottka I, Wegscheider K, Aepfelbacher M. Rapid identification of bacteria from positive blood culture bottles by use of matrix-assisted laser desorption-ionization time of flight mass spectrometry fingerprinting. J Clin Microbiol 2010; 48: 1584-91. 